\title{
Identification of pulsation modes from photometry
}

\author{
Michel Breger ${ }^{1,2}$ \\ ${ }^{1}$ Department of Astronomy, University of Texas, Austin, TX 78712, USA \\ email: breger@astro.as.utexas .edu \\ ${ }^{2}$ Institut für Astrophysik der Universität Wien, Türkenschanzstr. 17, A-1180, Wien, Austria
}

\begin{abstract}
The identification of the detected pulsation modes in terms of the spherical harmonic quantum numbers is crucial for asteroseismology. Light curves obtained in different passbands have become an important tool for mode identifications, which rely on wavelength-dependent amplitudes and phase shifts. We demonstrate this for different types of pulsators and review recent successes from earth-based measurements, especially in determining the important $l$ values. The extensive amount of accurate data needed to determine small phase shifts and accurate amplitude ratios suggests multicolor measurements using space satellites. This motivated the multicolor BRITE satellite project, for which the first two satellites have already been launched successfully. We demonstrate the potential from models computed for the BRITE wavelengths. Most of the excellent presently available satellite photometry is not multicolor, although frequencies with amplitudes as small as a few parts-per-million have been detected and confirmed. We briefly discuss mode identifications from frequency patterns, including the use of correlations between phase and amplitude changes.
\end{abstract}

Keywords. techniques: photometric, space vehicles, stars: oscillations (including pulsations), stars: evolution, stars: early-type, stars: $\delta$ Scuti

\section{Introduction}

The recent advances in high-precision photometry have led to the discovery of several hundreds of simultaneously present pulsation frequencies in a large number of individual stars. For space missions such as Kepler and CoRoT, the present detection threshold limits are near one part-per-million in amplitude. This represents an improvement of two orders of magnitude over previous earth-based telescope measurements.

To use the frequency data for asteroseismic modeling, the correct astrophysical identification of the detected frequency peaks is essential. This involves the identification of the nature of the frequency peaks (e.g., pulsation and the type of mode, rotation, granulation, nonlinear effects such as combination frequencies, magnetic field effects). In addition, for those peaks identified with pulsation modes, we need to identify the discrete spherical harmonic quantum numbers $(l, m, n)$ for as many of the detected oscillation modes as possible. The latter process is generally referred to as 'mode identification'. However, the recently obtained rich frequency data for many types of pulsators have shown that considerable attention also needs to be paid to the astrophysical identification of the frequency peaks, especially to those with small amplitudes. This is made possible by examining the regularities in the frequency spacings. Especially due to the extremely high frequency resolution of some spacecraft data, these regularities can be determined very precisely and lead to different families of frequencies, each associated with specific astrophysical properties of the star. 


\section{Mode identification from multicolor photometry}

During its pulsation cycle, a star changes in temperature, radius and geometrical crosssection. The relative changes depend on the type of pulsation mode (e.g., gravity and pressure modes) as well as the spherical harmonic quantum numbers. In principle, it is possible to separate the effects by studying the light variability as a function of wavelength (multicolor photometry), radial velocity and changes in the spectroscopic line-profiles. In practice, such information is available for only a few stars. Photometrically, the changes lead to wavelength-dependent amplitudes and times of light maxima. If we observe with two different filters, we will see an amplitude ratio and phase difference between the two passbands.

Already in 1977, in a pioneering paper, Dziembowski (1977) discussed the comparison of light and radial-velocity variations in order to derive the spherical harmonics of nonradial modes. Balona \& Stobie (1979) applied the method to the hot $\beta$ Cep stars to identify radial and quadrupole modes. Watson (1988) applied a 'modern' method of comparing amplitude ratios, $A_{B-V}$, and phase differences, $\phi_{B-V}-\phi_{V}$, derived from light curves taken through two passbands to the large-amplitude $\delta$ Scuti star AI Vel. He successfully identified the two observed modes to be radial.

During the last ten years, the method has been extended and applied to many types of pulsating stars by a large number of colleagues. Daszyńska-Daszkiewicz et al. (2003, $2005)$ introduced the nonadiabatic $f$-parameter, which is the ratio of the amplitude of the bolometric flux perturbations to the radial displacement. While the theoretical values of $f$ are obtained from stellar pulsation calculations, multicolor photometry together with radial-velocity measurements provide the empirical counterparts together with the $l$ value. A number of extensive discussions and summaries of the theoretical problems and results have become available in the last few years and we refer to an excellent review by Daszyńska-Daszkiewicz \& Pamyatnykh (2013). The possible sources of error in the theoretical values of amplitude ratios and phase differences of main-sequence B-type stars were discussed by Daszyńska-Daszkiewicz \& Szewczuk (2011). Some of the most complex seismic modelling for mode identification of a B-type pulsator $(\gamma$ Peg) was performed by Walczak et al. (2013).
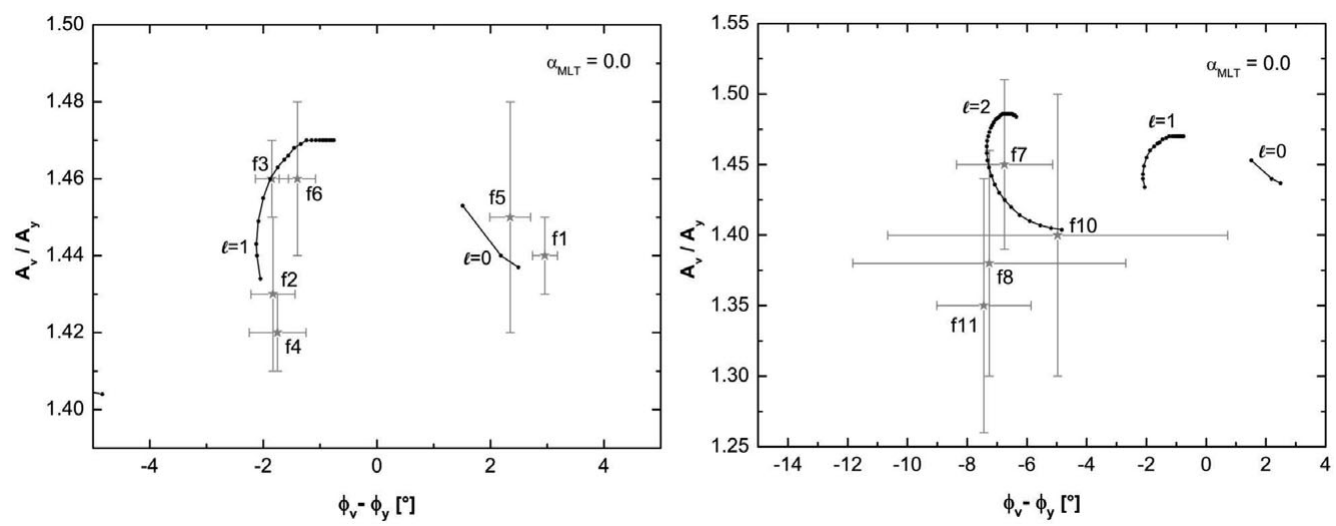

Figure 1. Amplitude ratios and phase differences in the $v$ and $y$ passbands for different pulsation modes of 44 Tau. The observationally well-determined values (for $l$ values of 0 and 1 ) are shown in the left panel, while the $l=2$ modes have larger uncertainties (after Lenz et al. 2008).

Let us illustrate the comparison between the amplitude ratio and phase difference observations with models for two extensively studied A/F stars. Figure 1 shows the 


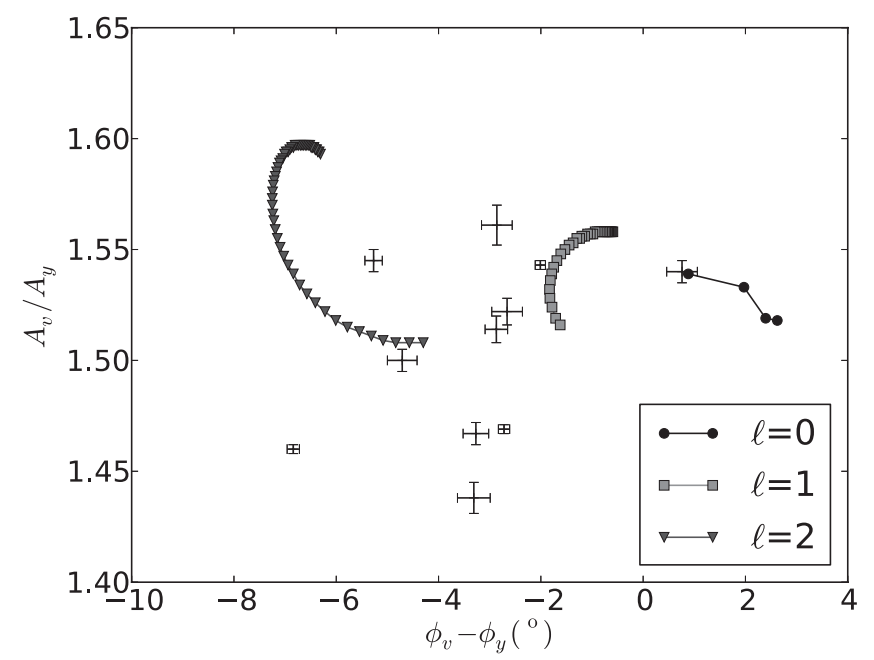

Figure 2. Amplitude ratios and phase differences in the $v$ and $y$ passbands for different pulsation modes of $4 \mathrm{CVn}$. The error bars are determined by comparing results from 702 nights covering eight different years and the results may be the most precise two-color values obtained so far. The comparison with a preliminary theoretical model (see text) covering the frequency range from 4.5 to $9.5 \mathrm{~d}^{-1}$ is also shown.

results for the extremely slow rotator 44 Tau (142 nights, Lenz et al. 2008), for which the $l$ values of the dominant pulsation modes could be successfully identified.

The evolved $\delta$ Scuti star $4 \mathrm{CVn}$ has been photometrically studied for more than 50 years. From 2005-2012, we obtained more than 700 nights of high-precision photometry of this star with the Vienna Automatic Photoelectric Telescope. The Strömgren $v$ and $y$ passbands were used. Figure 2 presents the amplitude ratios and phase differences for eleven dominant pulsation modes, together with preliminary models computed by P. Lenz and A.A. Pamyatnykh. The models used a mass of 2.1 solar masses, $\alpha=0.2$ and a metallicity of $[\mathrm{M} / \mathrm{H}]=+0.2$ to fit the radial mode. The small error bars of the observational data provide the opportunity to refine the input values used for the theoretical models computed for this star. We note here that the spectroscopic line-profile analyses agree with the photometric mode identifications.

\subsection{Are the formal uncertainties underestimated?}

The extensive multiyear data of $4 \mathrm{CVn}$ also allow us to examine whether the size of the formal errors computed from multiple-least-squares fits to the photometric observations (e.g., with PERIOD04, Lenz \& Breger 2005) are possibly underestimated. Such an underestimation could be caused by systematic (as opposed to random) errors in photometry. We have computed amplitude ratios and phase differences with their annual uncertainties for each of the years from 2005-2012. We then averaged the annual values of the amplitude ratios and phase shifts and compared the standard deviation of the averages with those expected from the annual values. The process was applied to the seven modes with the highest amplitudes. For the amplitude ratio we found that the annual results had a standard deviation of 0.82 of the expected value, while for the phase shifts a ratio of 1.12 was obtained.

We conclude that the formal least-squares errors computed for the amplitude ratios and phase shifts are confirmed. 


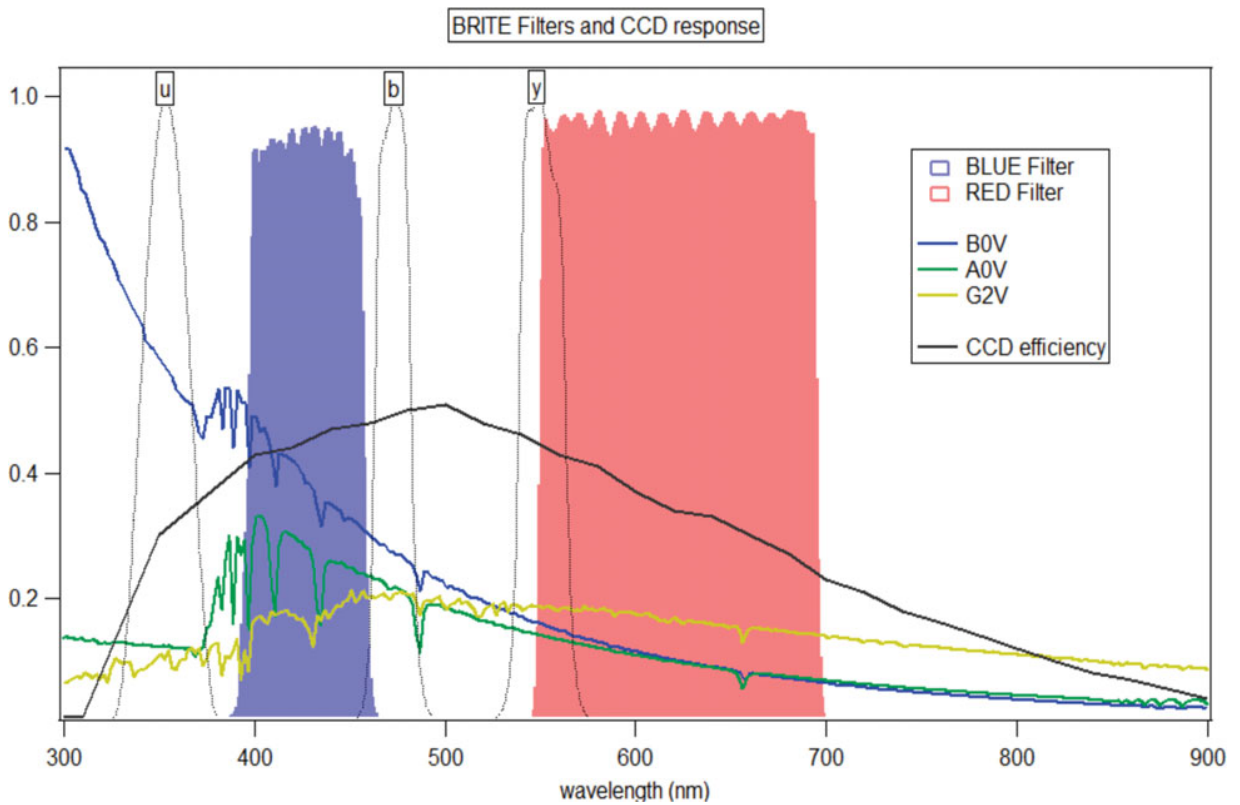

Figure 3. Transmissions of the blue and red interference filters together with the spectral energy distributions of $\mathrm{B} 0 \mathrm{~V}, \mathrm{~A} 0 \mathrm{~V}$ and $\mathrm{G} 2 \mathrm{~V}$ stars. The response of the CCD BRITE detector is also shown together with the positions of the Strömgren $u, b$ and $y$ passbands. (Diagram courtesy of Werner Weiss.)

\section{The multicolor BRITE satellites and earth-based radial-velocity measurements}

Recent space missions, such as Kepler, provide a quantum jump in our observational studies of stellar pulsation. These space observations are single-color, which means that multicolor mode identification is not possible. This provides one of the main motivations for the BRITE nanosatellite mission and we refer to an extensive discussion in another paper in this volume. The BRITE telescopes have small apertures of $3 \mathrm{~cm}$ and are therefore limited to observing bright stars. Based on photon-statistics calculations, the amplitude ratios and phase shifts expected for the BRITE nanosatellites should nevertheless be accurate and enable mode identifications.

Figure 3 shows the transmissions of the blue and red interference filters.

The chosen filters provide an excellent separation of the modes with different $l$ values in the amplitude ratio, phase difference diagram. This is illustrated in Fig. 4, which shows the results of computations by J. Daszyńska-Daszkiewicz. The SPB model uses $T_{\text {eff }}=$ $16000 \mathrm{~K}, \log L / L_{\odot}=3.2$ and a mass of 6 solar masses, while the RR Lyrae star model was computed with a mass of 0.63 solar masses, $T_{\text {eff }}=6540 \mathrm{~K}$, and $\log L / L_{\odot}=1.68$, and $\alpha_{\text {conv }}=0.0$. Both models use OP opacities with $X=0.74, Z=0.0006$.

\subsection{Why earth-based radial-velocity measurements should be simultaneous}

The BRITE project uses two (rather than three or more) passbands. Consequently, additional radial velocity measurements from the ground are highly recommended to eliminate many of the remaining uncertainties of the pulsation models used for mode identifications. We would like to stress that such measurements should be nearly simultaneous with the space measurements and not be spaced, say, six months apart due to the limited accessibility of stars observed from the ground. The reason is the fact that 

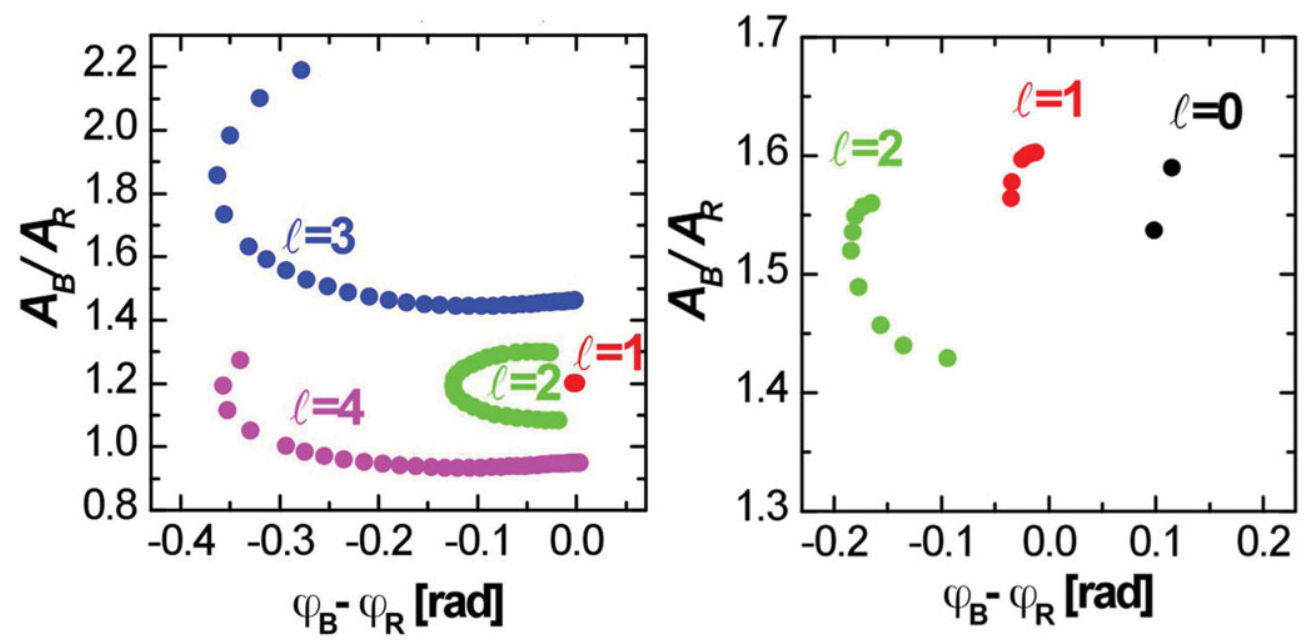

Figure 4. Predicted amplitude ratios and phase differences in the BRITE $B$ and $R$ passbands for different pulsation modes of a SPB (left panel) and a RR Lyrae star model (right panel). Note the clear separations of the different $l$ values. (Models and diagrams courtesy of J. Daszyńska-Daszkiewicz.)

Table 1. The case for simultaneous photometric/radial-velocity data: Observed phase shifts in two $\delta$ Scuti stars over 6 months.

\begin{tabular}{lrrl}
\hline Star & Mode & $\begin{array}{c}\text { Phase shift } \\
\text { cycles }\end{array}$ & Comments \\
& & & \\
\hline 4 CVn & $7.38 \mathrm{~d}^{-1}$ & $0.082 \pm 0.006$ & Steady change 2005-2012, 702 telescope nights \\
KIC 8054146 & $25.95 \mathrm{~d}^{-1}$ & $0.086 \pm 0.002$ & Relatively stable mode, 3 years SC Kepler data \\
\hline
\end{tabular}

nonradial pulsation modes show small, steady amplitude and period changes. These accumulate over several months to such an extent that the correct phase shifts between radial velocities and photometry can no longer be determined.

Let us illustrate this point by using presently unpublished, recent analyses of two wellstudied stars: $4 \mathrm{CVn}$ with a total of over 700 nights of ground-based observations from 2005 to 2012, and KIC 8054146, studied continuously by the Kepler spacecraft for three years. Both stars are pulsators of spectral types A/F, but the argument should apply to B stars as well. In both stars, all observed modes show slow, systematic period changes. For each star, we pick a typical, stable mode of relatively high amplitude and calculate the phase changes over six months. We note again that these stars were observed for many years and that the results are relatively independent of the choice of six-month time period. The results are shown in Table 1. They indicate that the photometric and spectroscopic measurements should not be spaced months apart.

\section{Mode identification from frequency patterns}

Recent space measurements of pulsating stars have revealed the existence of hundreds of frequency peaks in individual stars. Since these space missions have been single-color, multicolor mode identification has not been possible. Consequently, the examination of frequency patterns, amplitude variability and lifetime determinations of individual 


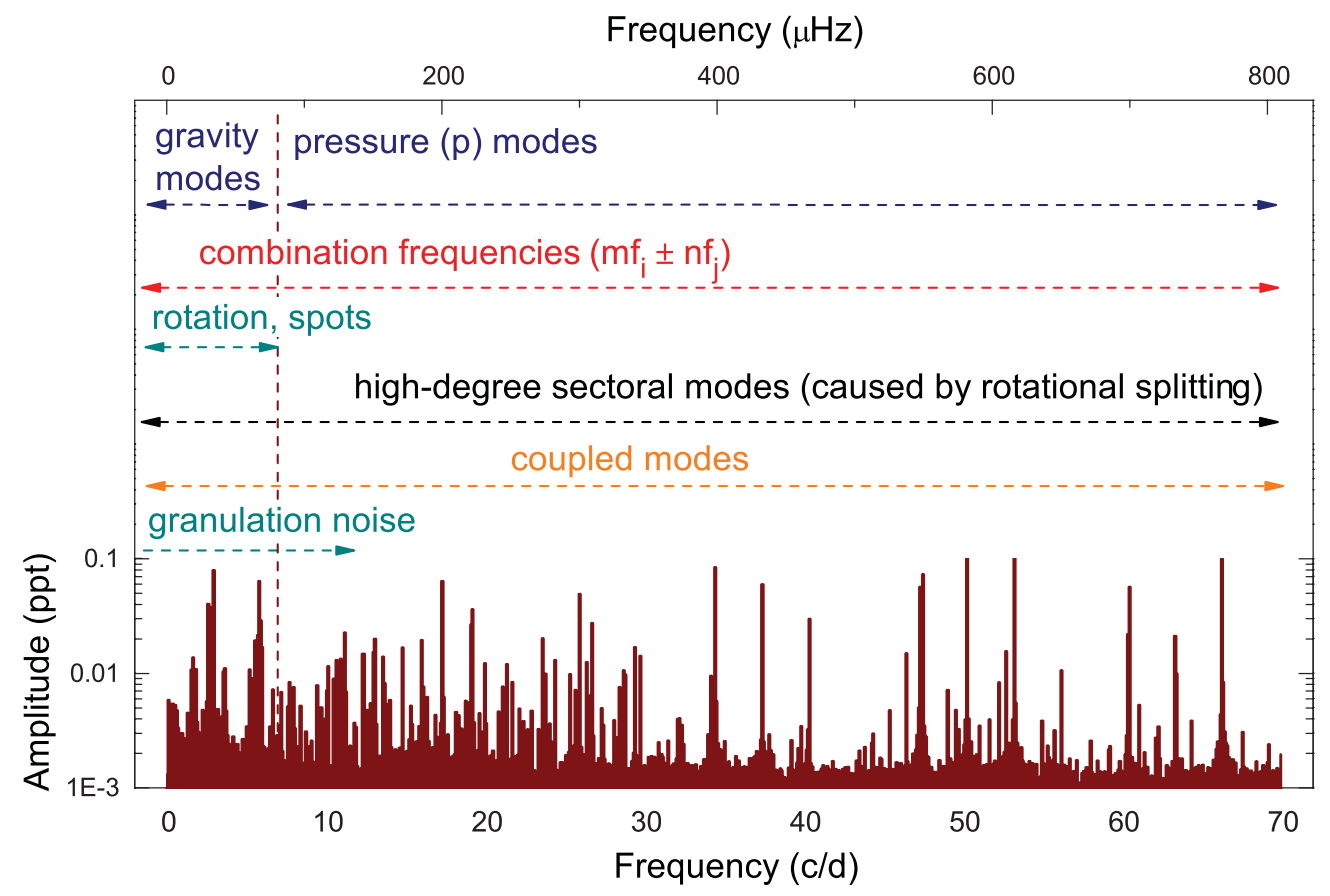

Figure 5. Different astrophysical origins of detected frequencies in main-sequence and slightly evolved A stars. While the frequencies and amplitudes of KIC 8054146 (Breger et al. 2012) are given as an example, the interpretations should fit other B, A and F stars as well.

frequencies have become the major tools of analysis. A successful application of this method of mode identification was the discovery of 24 gravity modes of the same $l$ degree through equal period-spacing in CoRoT ID 105733033 by Chapellier et al. (2012).

Figure 5 presents a non-exhaustive list of different astrophysical origins in different frequency regions. It demonstrates that the astrophysical origin of each frequency peak needs to be examined carefully.

Combination frequencies and harmonics: Multiple pulsation modes lead to a number of nonlinear interaction terms of the form $\left(m f_{1}+n f_{2}\right)$, where $f_{1}$ and $f_{2}$ are the pulsation frequencies, and $m$ and $n$ are integers. The amplitudes of these combination frequencies are generally much smaller than those of $f_{1}$ and $f_{2}$. Nevertheless, at the amplitude level of several parts-per-million, as many as hundreds of combination frequencies can be seen. Therefore, it becomes extremely important to separate these from independent pulsation modes by using data with high frequency resolution (i.e., long data sets).

Rotation: Kepler observations of A stars, analyzed by Balona (2011), have revealed that the distribution of dominant low frequencies match the expected distribution for rotational frequencies. In fact, for KIC 9700322, the unusually slow rotation suggested by the low-frequency peak at $0.16 \mathrm{~d}^{-1}$ was confirmed by an HET spectrum showing $v \sin i=19 \pm 1 \mathrm{~km} \mathrm{~s}^{-1}$ and the spacing of an $l=2$ quintuplet (Breger et al. 2011). Due to differential rotation, spots can also lead to several low-frequency peaks.

Granulation noise: Kallinger \& Matthews (2010) have argued that even in stars of spectral type A, a large number of low-amplitude peaks in the power spectrum are actually granulation noise.

High-degree sectoral gravity modes: Due to rotational splitting, in the observer's frame of reference, high-degree sectoral modes are shifted to high frequencies with equidistant 


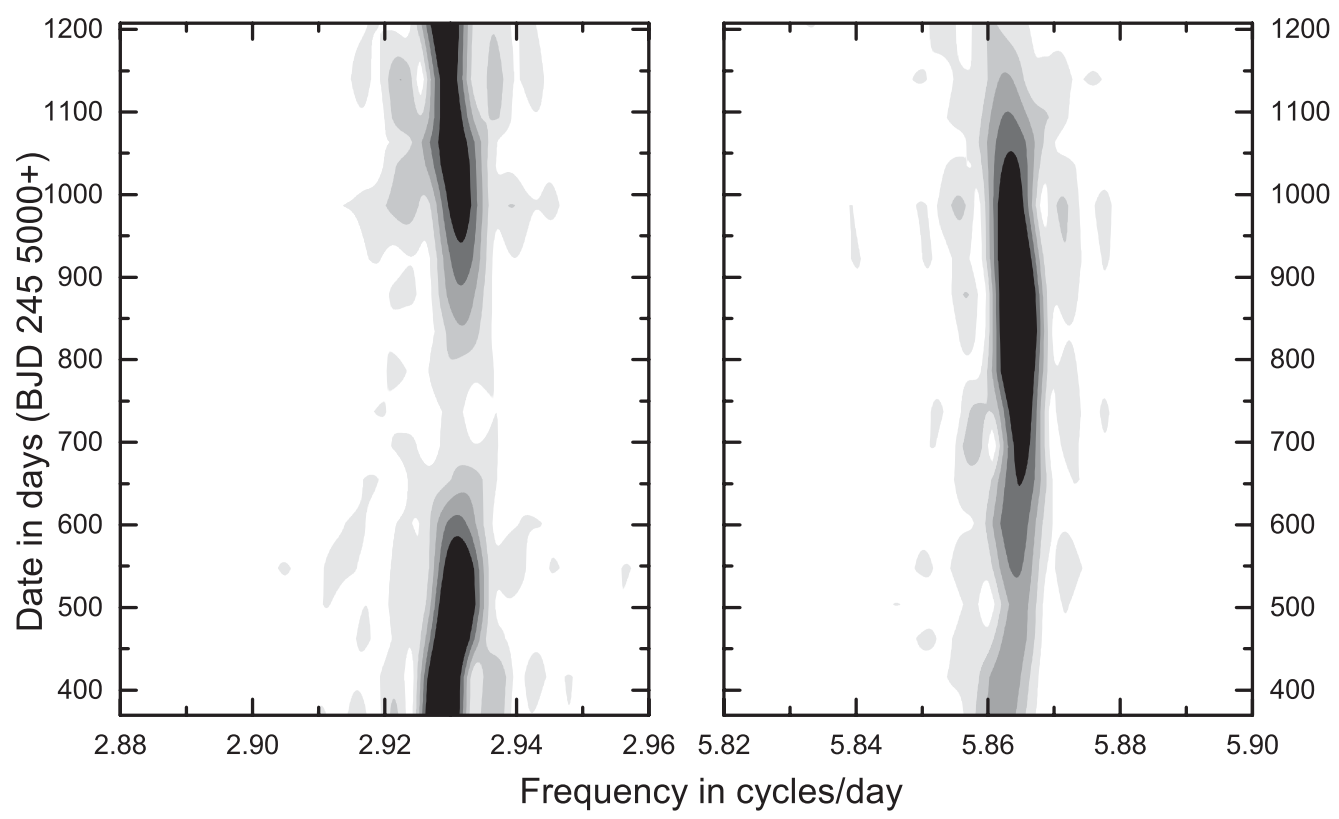

Figure 6. Fourier time plots covering three years for the 2.93 and $5.86(=2 \times 2.93) \mathrm{d}^{-1}$ frequencies of KIC 8054146. Both frequencies have strongly variable amplitudes of similar sizes. The variations are, however, not connected. This is seen in other main-sequence stars as well and demonstrates that the higher frequency is not a $2 f$ harmonic of the lower frequency. The amplitude variations of both frequencies can be correctly predicted by mode coupling of specific high frequencies.

spacing. This was already shown by Kennelly et al. (1998) during their spectroscopic MUSICOS multisite project for a number of $\delta$ Scuti stars such as $\tau$ Peg. Recently, prograde sectoral modes have also been reported for Rasalhague (Monnier et al. 2010) and KIC 8054146 (Breger et al. 2013).

\section{Those pesky $f$ and $2 f$ low-frequency peaks}

A large number of stars of spectral type B, A and F studied by the Kepler spacecraft show low frequencies with an apparent harmonic term, i.e., $f$ and $2 f$. Could these peaks be pulsation and what are their mode identifications? A conventional explanation of the $2 f$ term would be the Fourier expansion of a nonsinusoidal light curve associated with $f$. It would then be ignored for mode identification or the determination of the rotational velocity. However, in a number of $\mathrm{A} / \mathrm{F}$ stars, the $2 f$ term has amplitudes as large as those of the frequency $f$. Furthermore, both the $f$ and $2 f$ low frequencies are also preferred spacings of high-frequency p modes. This challenges the explanation in terms of harmonics.

For many of these stars, several years of continuous photometric data are available. This allows us to examine the amplitude and phase variations of the different peaks. For the star KIC 8054146 these variations are considerable. In Fig. 6, we show the Fourier time plots of the 2.93 and $5.86 \mathrm{~d}^{-1}$ frequency peaks, which use the same amplitude scale. The amplitude variations are not similar, which does not support the Fourier harmonics theory. In fact, mode-coupling by high-frequency p modes can correctly predict the amplitude variations of these low-frequency peaks to a few parts-per-million. We note here that the star is a rapidly rotating star, for which mode coupling is expected. 


\section{Conclusion}

In this short review, we summarized recent developments of mode identification from photometry. We emphasized that with the advent of extremely high-precision spacecraft data, the first step of mode identification is to separate the independent pulsation modes from the multitude of additional frequency peaks. While the recognition of specific frequency patterns in the amplitude vs. frequency diagram has been successful in a number of cases, we require multicolor photometry in order to determine the discrete spherical harmonic quantum numbers $(m, l, n)$ for as many of the detected oscillation modes as possible.

Such multicolor photometry will be provided by the recently launched BRITE satellites. These measurements should be accompanied by near-simultaneous radial-velocity measurements. Since the majority of stars to be studied are fast rotators, the observations will also provide valuable input for the inclusion of fast rotation into the present stellar models.

\section{Acknowledgements}

This investigation has been supported by the Austrian Fonds zur Förderung der wissenschaftlichen Forschung through project P21830-N16.

\section{References}

Balona, L. A. 2011, MNRAS, 415, 1691

Balona, L. A. \& Stobie, R. S. 1979, MNRAS, 189, 649

Breger, M., Balona, L., Lenz, P., et al. 2011, MNRAS, 414, 1721

Breger, M., Fossati, L., Balona, L., et al. 2012, ApJ, 759, 62

Breger, M., Lenz, P., \& Pamyatnykh, A. A. 2013, ApJ, 773, 56

Chapellier, E., Mathias, P., Weiss, W. W., Le Contel, D., \& Debosscher, J. 2012, A\& $A, 540$, A117

Daszyńska-Daszkiewicz, J. \& Pamyatnykh, A. A. 2013, in: J. C. Suarez, R. Garrido, L. A. Balona \& J. Christensen-Dalsgaard (eds.), Stellar Pulsations, Astrophysics and Space Science Proceedings, Vol. 31 (Berlin: Springer), p. 179

Daszyńska-Daszkiewicz, J. \& Szewczuk, W. 2011, ApJ, 728, 2011

Daszyńska-Daszkiewicz, J., Dziembowski, W., \& Pamyatnykh, A. A. 2003, A\& A, 407, 999

Daszyńska-Daszkiewicz, J., Dziembowski, W., \& Pamyatnykh, A. A. 2005, A\&SA, 441, 641

Dziembowski, W. 1977, AcA, 27, 203

Kallinger, T. \& Matthews, J. M. 2010, ApJ, 711, L35

Kennelly, E. J., Brown, T. M., Kotak, R., et al. 1998, ApJ, 495, 440

Lenz, P. \& Breger, M. 2005, CoAst, 146, 53

Lenz, P., Daszyńska-Daszkiewicz, J., Pamyatnykh, A. A., \& Breger, M. 2008, CoAst, 153, 40

Monnier, J. D., Townsend, R. H. D., Che, X., et al. 2010, ApJ, 725, 1192

Walczak, P., Daszyńska-Daszkiewicz, J., Pamyatnykh, A. A., \& Zdravkov, T. 2013, MNRAS, 432,822

Watson, R. D. 1988, Ap\&SS, 140, 255 